\title{
Establishing evidenced-based best practice for the de novo assembly and evaluation of transcriptomes from non-model organisms
}

\author{
Matthew D. MacManes ${ }^{1}$, \\ 1 Department of Molecular, Cellular and Biomedical Sciences, University of New Hampshire, Durham NH, \\ USA \\ * E-mail: macmanes@gmail.com \\ $\oplus$ Twitter: @macmanes
}

\section{Abstract}

Characterizing transcriptomes in both model and non-model organisms has resulted in a massive increase in our understanding of biological phenomena. This boon, largely made possible via high-throughput sequencing, means that studies of functional, evolutionary and population genomics are now being done by hundreds or even thousands of labs around the world. For many, these studies begin with a de novo transcriptome assembly, which is a technically complicated process involving several discrete steps. Each step may be accomplished in one of several different ways, using different software packages, each producing different results. This analytical complexity begs the question - Which method(s) are optimal? Using reference and non-reference based evaluative methods, I propose a set of guidelines that aim to standardize and facilitate the process of transcriptome assembly. These recommendations include the generation of between 20 million and 40 million sequencing reads from single individual where possible, error correction of reads, gentle quality trimming, assembly filtering using Transrate and/or gene expression, annotation using dammit, and appropriate reporting. These recommendations have been extensively benchmarked and applied to publicly available transcriptomes, resulting in improvements in both content and contiguity. To facilitate the implementation of the proposed standardized methods, I have released a set of version controlled open-sourced code, The Oyster River Protocol for Transcriptome Assembly, available at http://oyster-river-protocol.rtfd.org/. 


\section{${ }_{18}$ Introduction}

For all biology, modern sequencing technologies has provided for an unprecedented opportunity to gain a deep understanding of genome level processes that underlie a very wide array of natural phenomenon, from intracellular metabolic processes to global patterns of population variability. Transcriptome sequencing has been influential, particularly in functional genomics, and has resulted in discoveries not possible even just a few years ago. This in large part is due to the scale at which these studies may be conducted. Unlike studies of adaptation based on one or a small number of candidate genes (e.g. (Fitzpatrick et al., 20105; Panhuis, (2006)), modern studies may assay the entire suite of expressed transcripts - the transcriptome simultaneously. In addition to issues of scale, as a direct result of enhanced dynamic range, newer sequencing studies have increased ability to simultaneously reconstruct and quantitate lowly- and highly-expressed transcripts, (Vijay et al., 2013; Wolt, 2013). Lastly, improved methods for the detection of differences in gene expression (e.g., (Love et al, [2014; Robinson et al, 2010)) across experimental treatments has resulted in increased resolution for studies aimed at understanding changes in gene expression.

As a direct result of their widespread popularity, a diverse toolset for the assembly and analysis of transcriptome exists. Notable amongst the wide array of tools include several for quality visualization FastQC (available here) and SolexaQA (Cox et al, 2010), read trimming (e.g. Skewer (Jiang et al., 2014), Trimmomatic (Bolger et al., 2014) and Cutadapt (Martin, 2011)), read normalization (khmer (Pell et all, 2012)), error correction (Le et al, [2013), assembly (Trinity (Haas et al., 2013), SOAPdenovoTrans (Xie et al., [2014)), and assembly verification (Transrate (Smith-Unna et al, 2015)), BUSCO (Benchmarking

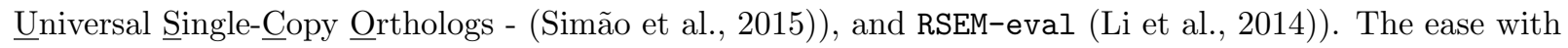
which these tools may be used to produce transcriptome assemblies belies the true complexity underlying the overall process. Indeed, the subtle (and not so subtle) methodological challenges associated with transcriptome reconstruction may result in highly variable assembly quality. Amongst the most challenging include isoform reconstruction and simultaneous assembly of low- and high-coverage transcripts (Johnson et al, 2010.3; Modrek et al, 2010I), which together make accurate transcriptome assembly technically challenging. Production of an accurate transcriptome assembly requires a large investment in time and resources. Each step in it's production requires careful consideration. Here, I propose a set of evidence-based guidelines for assembly and evaluation that will result in the production of the highest quality transcriptome assembly possible.

Currently, a very large number of labs and research programs depend, often critically, on the production of accurate transcriptome resources. That no current best practices exits - particularly for those working in 
non-model systems - has resulted in an untenable situation where each laboratory makes up it's own computational pipeline. These pipelines, often devoid of rigorous quality evaluation, may have important downstream consequences. This manuscript, by proposing a specific evidence-based process, significantly enhances the technical quality and reproducibility of transcriptome studies, which is critical for this emerging field of research.

\section{Methods}

To demonstrate the merits of my recommendations, a large number of assemblies were produced using a variety of methods. For all assemblies performed, Illumina sequencing adapters were removed from both ends of the sequencing reads, as were nucleotides with quality Phred $\leq 2$, using the program Trimmomatic version 0.32 (Bolger et al., 2014). The reads were assembled using Trinity release 2.1.1 (Haas et al., 20133) using default settings. Trinity was used as the default assembler as it has been previously reported to be best in class (Li et al, 2014; Smith-Unna et al., 2015). Assemblies were characterized using Transrate version 1.0.1 (Smith-Unna et al, [2015). Using this software, I generated three kinds of metrics: contig metrics; mapping metrics which used as input the same reads that were fed into the assembler for each assembly; and comparative metrics which used as input the Mus musculus version 75 transcriptome. In addition to the metrics provided by Transrate, I evaluated completeness of each assembly by use of BUSCO, a software package that searches for highly conserved, near-universal, single copy orthologs. All assemblies generated are available here, and will be moved to Dryad on acceptance.

To understand the influence of read depth on assembly quality, I produced subsets of size 1,2,5,10,20,40,60,80,100 million paired end reads of two publicly available paired-end datasets - A Mus dataset -SRR797058 described in (Han et al, [2113) and a human dataset - SRR1659968. The subsampling procedure was accomplished via the software package seqtk (https://github.com/lh3/seqtk). For the evaluation of the effects of sequence polymorphism on assembly quality, I use reads from BioProject PRJNA157895 described in (MacManes and Lacey], 2012), a Ctenomys dataset which consists of 10 read files from the hypothalami of 10 different individuals. This dataset was assembled two ways. First, the reads from all 10 individuals were jointly assembled in one large assembly $\llbracket \mathrm{CODE} \|$. This assembly was compared to the assembly of a single individual $\llbracket \mathrm{CODE} \mid$. Assemblies were generated and evaluated as per above.

To evaluate the effects of error correction, I used the subsampled read datasets, which were subsequently error corrected using the following software packages: SEECER version 0.1.3 (Le et all, 20113), Lighter version 1.0.7 (Song et al., 2014), SGA version 0.10.13 (Simpson and Durbin, 2012), bf c version r177 (Lil, 
2015), RCorrector (Song and Florea, 2015), and BLESS version 0.24 (Heo et aL, 2014). In correction algorithms (SGA, BLESS, bfc) that allowed for the use of larger kmer lengths, I elected to error correct with a small $(k=31)$ and a long $(k=55) k m e r$, while for the other software (RCorrector, SEECER and Lighter) that does not allow for longer $k m e r$ values, I set $k=31$. bfc requires interleaved reads, which was accomplished using khmer version 2.0 (Alameldin et al., 2015; Brown et al., 2012; McDonald and Brown, 2013). Code for performing these steps is available $\|$ here .

The effects of khmer digital normalization (एell et al., 2012) were characterized by generating three 20 million and three 100 million read subsets of the larger Mus dataset. Digital normalization was performed using a median kmer abundance threshold of 30. The resulting datasets were assembled using Trinity, and evaluated using BUSCO and Transrate. Code for performing these steps is available in the diginorm target of the Makefile\|.

Post-assembly processing was evaluated using several assembly datasets of various sizes, generated above. Each assembly was evaluated using Transrate. Transrate produces a score based on contig and mapping metrics, as well as a more optimal assembly where poorly supported contigs (putative assembly artifacts) are removed. Both the original and Transrate optimal assembly are evaluated using BUSCO, to help better understand if filtration results in the loss of non-artifactual transcripts. In addition to Transrate filtration, an additional, or alternative filtration step is performed using estimates of gene expression (TPM=transcripts per million). TPM is estimated by two different software packages that implement two distinct methods - Salmon (Patro et al., 2015) and Kallisto (Bray et al., 2015). Transcripts whose expression is estimated to be greater than a given threshold, typically TPM=1 or TPM=0.5 are retained. As above, the filtered assemblies are evaluated using BUSCO, to help better understand if filtration results in the loss of non-artifactual transcripts. Code for performing these steps is available in the QC target of the makefile available $\|$ here\|.

\section{Recommendations}

\subsection{Input Data}

\section{Summary Statement: Sequence 1 or more tissues from 1 individual to a depth of between 20} million and 40 million $100 \mathrm{bp}$ or longer paired-end reads.

\footnotetext{
When planning to construct a transcriptome, the first question to ponder is the type and quantity of
} 
data required. While this will be somewhat determined by the specific goals of the study and availability of tissues, there are some general guiding principals. As of 2014, Illumina continues to offer the most flexibility in terms of throughout, analytical tractability, and cost (Glenn, [201) and as a result, the recommendations here are primarily related to assembly using Illumina data. It is worth noting however, that long-read (e.g. PacBio) transcriptome sequencing is just beginning to emerge as an alternative (Au et al, [2013), particularly for researchers interested in understanding isoform complexity. Though currently lacking the throughput for accurate quantitation of gene expression, long read technologies, much like they have done for de novo genome assembly, seem likely to replace short-read-based de novo transcriptome assembly at some point in the future.

For the typical transcriptome study, one should plan to generate a reference based on 1 or more tissue types, with each tissue adding unique tissue-specific transcripts and isoforms. Though increasing the amount of sequence data collected does increase the accuracy and completeness of the assembly (Figure 1, 3) albeit marginally, a balance between cost and quality exists. For the datasets examined here (vertebrate tissues), sequencing more than between $20 \mathrm{M}$ and $40 \mathrm{M}$ paired-end reads is associated with the discovery of very few additional transcripts, and only minor improvement in other assembly metrics. Read length should be at least $100 \mathrm{bp}$, with longer reads likely aiding in isoform reconstruction and contiguity (Garber et al, 2011). In the case where multiple tissues are sequenced, it is likely best to combine reads from each tissue together to produce a joint assembly.

Because sequence polymorphism increases the complexity of the de bruijn graph (Iqbal et al., 2012; Studholme, [2010), and therefore may negatively effect the assembly itself, the reference transcriptome should be generated from reads corresponding to as homogeneous a sample as possible. For outbred, non-model organisms, this usually means generating reads from a single individual. When more then one individual is required to meet other requirements (e.g., for differential expression replicates or experimental treatment conditions), keeping the number of individuals to a minimum is paramount. For instance, when performing an experiment where a distinct set of genes may be expressed in different treatments (or sexes), the recommendation is to sequence one individual from each treatment class.

To illustrate this effect, I examined the effects of assembling reads from 10 individuals jointly, versus assembling a representative individual. This individual was selected based on having the highest number of reads. Using 30 threads on a standard Ubuntu workstation, the individual assembly of 38 million paired end read took approximately 23 hours and 20Gb of RAM, while the joint assembly took five days and 150Gb of RAM. In addition to this, to eliminate the potential confounding factor of increased coverage, I assembled another dataset that consisted of a random subsample of $3.8 \mathrm{M}$ paired-end reads from each of the 10 samples. 
This subsampled assembly used similar resources as did the single-individual assembly. Per Table 1, the joint assembly used more than eight times more reads, and is more than four times larger than the assembly of a single individual. Despite the additional read data, the Transrate score is markedly decreased, although the BUSCO statistics are slightly better. The large joint assembly suffers from major structural problems that are unfixable via the proposed filtering procedures. Specifically, read-mapping data suggests that $28.7 \%$ of the contigs in the joint assembly and $18 \%$ in the subsampled assembly could be merged, versus $15 \%$ in the single assembly. This structural problem is likely the result of sequence polymorphism and may cause significant issues for many common downstream processes.

\begin{tabular}{llllll} 
Name & Num. Reads & Num. Contigs & Assembly Size & Score & BUSCO \\
\hline Single Ind. & $38 \mathrm{M}$ & 205812 & $131.6 \mathrm{Mb}$ & 0.3064 & C:81\%,D:41\%,M:9\% \\
Subsampled & $38 \mathrm{M}$ & 304162 & $183.8 \mathrm{Mb}$ & 0.2619 & C:84\%,D:47\%,M:8.4\% \\
10 Ind. & $269 \mathrm{M}$ & 913295 & $440.2 \mathrm{Mb}$ & 0.22011 & C:88\%,D:51\%,M:5\% \\
\hline
\end{tabular}

Table 1. A comparison of the raw assemblies resulting from a single individuals versus the joint assembly of 10 individuals as well as a joint - but subsampled dataset consisting of a random $3.8 \mathrm{M}$ reads from each of the 10 samples. The individual assembly of 38 million reads resulted in a high quality assembly as evidenced by the Transrate score of 0.3064 (per (Smith-Unna et al., 2015), this is a score better that $50 \%$ of published transcriptomes), and a high BUSCO score. The assembly of 10 individuals scores lower using Transrate, though a small number of new transcripts are discovered. The joint-subsampled assembly recovered an equivalent number of transcripts, but resulted in a lower Transrate score, indicating a lower quality assembly. 


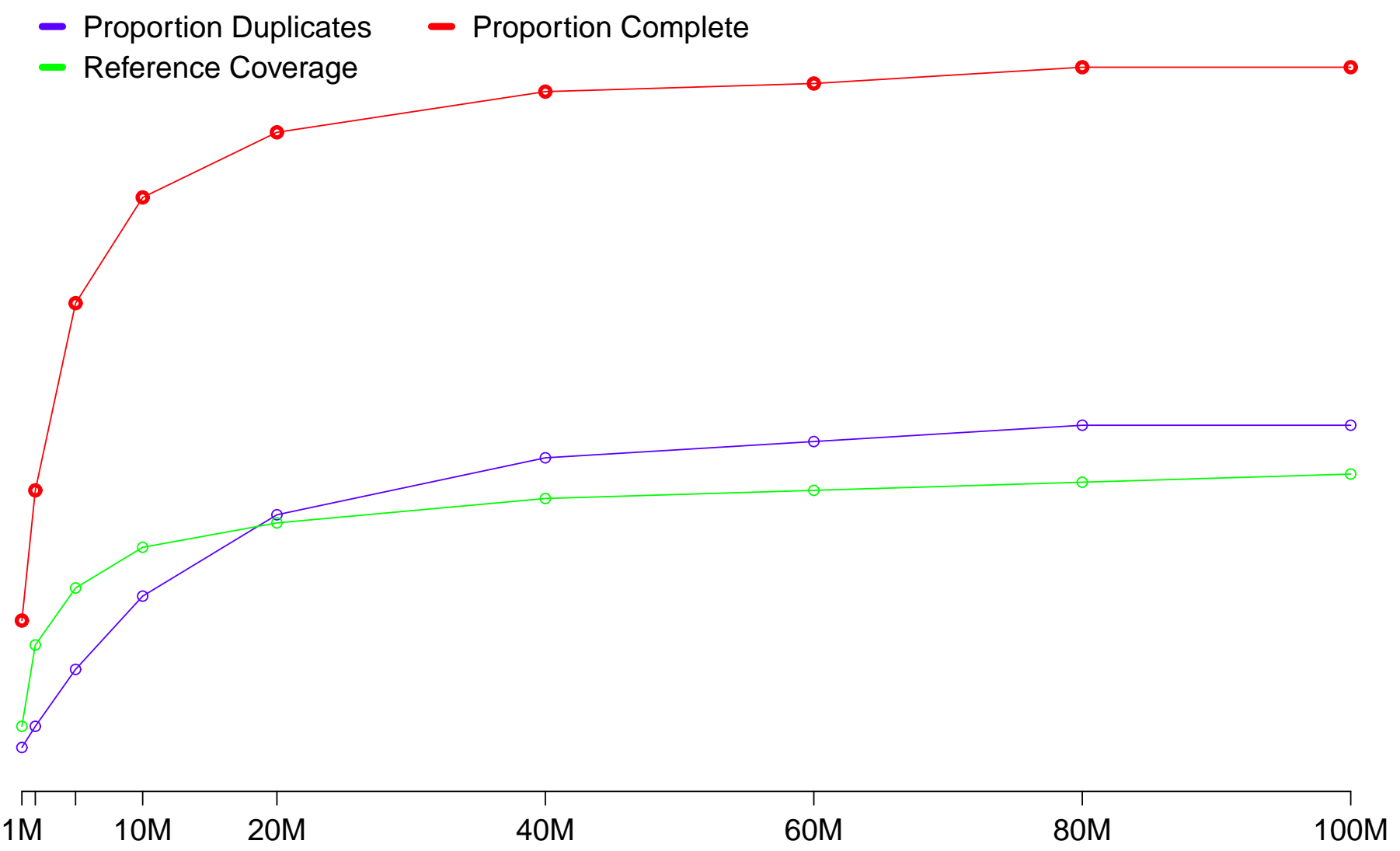

\title{
Number of reads
}

\begin{abstract}
Figure 1. Assembly of multiple subsetted datasets suggests that sequencing beyond 20-40 million paired end reads does not results in further sequence discovery. Proportion complete indicates the proportion of BUSCOs that were found to be full length. Proportion duplicates are those BUSCOs that were found multiple times in the assembly dataset. Reference coverage is a Transrate generated metric indicating the proportion of the reference Mus transcriptome found in the de novo assembly. Higher numbers for reference coverage and proportion complete indicate a more complete assembly.
\end{abstract}

\subsection{Quality Control of Sequence Read Data}

Summary Statement:Visualize your read data. Error correct reads using bfc for low to moderately sized datasets and RCorrector for higher coverage datasets. Remove adapters, and 
employ gentle quality filtering using PHRED $\leq 2$ as a threshold.

Before assembly, it is critical that appropriate quality control steps are implemented. It is often helpful to generate some metrics of read quality on the raw data. Several software packages are available - I am fond of SolexaQA (Cox et al, 2010$)$ and FastQC. Immediately upon download of the read dataset from the sequence provider, metrics of read quality, generated by either of these two software packages, should be generated. Of note - a copy of the raw reads should be compressed and archived, preferably on a physically separated device for long term archival storage. For this, I have successfully used Amazon S3 cloud storage, though many options exist.

Immediately after visualizing the raw data, error correction of the sequencing reads should be done (MacManes and Eisen, 2013). A very large number of read correction software packages exist, and several of them are benchmarked here using the Mus (Figure 2, and Tables S1-S11) and Homo datasets (Tables S12-S21). In all evaluated datasets, the error correction bfc was the best when correcting less than approximately 20M paired-end reads. When correcting more, the software RCorrector provided the optimal correction. The effects of error correction on assembly were evaluated using BUSCD and Transrate. While error correction did not result in significant improvements in BUSCO metrics, the transrate scores were substantially improved (Figure 3). These scores were largely improved by the fact that assemblies using error corrected reads had fewer low-covered bases and contigs, and a slightly higher mapping rate.

The error corrected reads are then subjected to vigorous adapter sequence removal, typically using Trimmomatic (Bolger et al., 2014) or Skewer (Jiang et al., 2014). With adapter sequence removal may be a quality trimming step. Here, substantial caution is required, as aggressive trimming has detrimental effects on assembly quality. Specifically, I recommend trimming at Phred=2 (MacManes, 2014), a threshold associated with removal of only the lowest quality bases. After adapter removal and quality trimming, the previously error corrected reads are now ready for de novo transcriptome assembly. 
Figure 2

๙

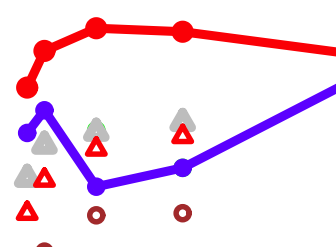

$\circ$

$\Delta$

$\Delta$

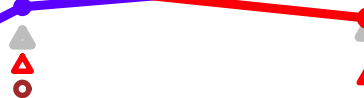

$\Delta \Delta$

$\Delta$

$\circ$

88.8

$\circ$

8

$\Delta$

$\stackrel{\circ}{\Delta}$

$\Delta$

$\stackrel{8}{\Delta}$

$\stackrel{\Delta}{\mathbf{Q}}$

$\triangle$

$\Delta$

$\stackrel{0}{\stackrel{0}{i}}$

$$
\begin{array}{ll}
\text { - bfc } & \text { - RCorrector } \\
\text { - SEECER } & \text { - Bless } \\
\text { - Lighter } & \text { SGA }
\end{array}
$$

\begin{tabular}{r|rr|rrr|r}
\hline 1 & 1 & & & & \\
$1 \mathrm{M}$ & $10 \mathrm{M}$ & $20 \mathrm{M}$ & $40 \mathrm{M}$ & $60 \mathrm{M}$ & $80 \mathrm{M}$ & $100 \mathrm{M}$
\end{tabular}

Number of reads

Figure 2. Error correction of reads results in a performance gain (defined as: (perfect error corrected reads - perfect raw reads) + reads made better - reads made worse). Perfect reads are reads that map to the reference without mismatch. Better and worse reads are those that map with fewer or more mismatches. Low coverage datasets are best corrected with bfc, which higher coverage datasets are optimally corrected with RCorrector. The best performing corrections improve the quality of more than $15 \%$ of reads. To emphasize the patterns of performance of the two best-performing error correctors, their points are connected by lines. 
Figure 3

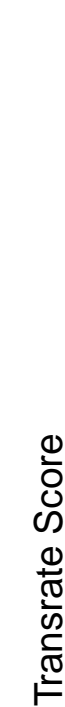

足

ֻே.

ִֻ

- Corrected reads

尺े

o

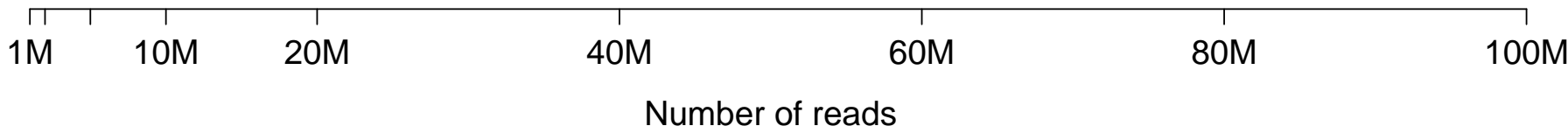

\section{- Raw reads}

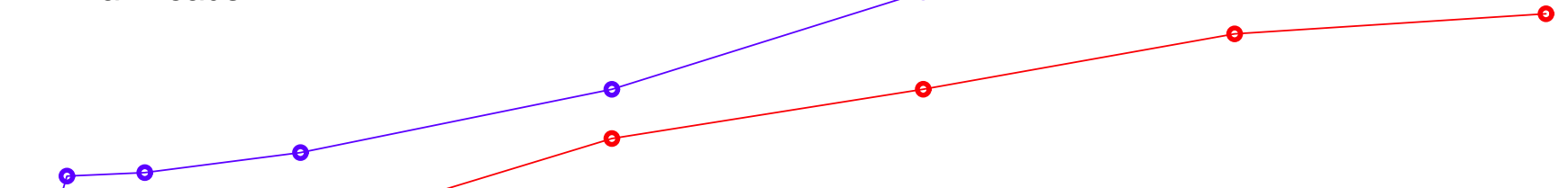

1

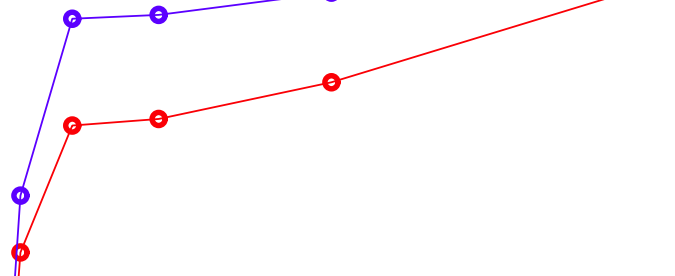

Number of reads

Figure 3. Error correction (with the best performing correction software, described in Figure 2), results in a consistent increase in the Transrate score, which indicates a higher quality assembly across all coverage depths. 


\subsection{Coverage normalization}

Summary Statement: Normalize your data, only if you have to.

Depending on the volume of input data, the availability of a high-memory workstation, and the rapidity with which the assembly is needed, coverage normalization may be employed. This process, which, using a streaming algorithm and measurement of the median kmer abundance of each read, aims to erode areas of high coverage while leaving untouched, reads spanning lower coverage areas. Normalization may be accomplished in the software package khmer (Pell et al, 2012), or within Trinity using a computational algorithm based on khmer. To evaluate khmer normalization, I generated 6 datasets, 3 using $20 \mathrm{M}$ paired reads and 3 using 100M paired reads. These datasets were assembled (available here) using Trinity and were evaluated via standard methods. These tests revealed that normalization did dramatically reduce RAM requirements and runtime, though it also decreased the number of complete BUSCO's found by an average of $1 \%$ for all assemblies. Normalization also decreased the Transrate score on average by 0.0248 for the $20 \mathrm{M}$ read assemblies and 0.0272 for the $100 \mathrm{M}$ read assemblies. Interestingly, normalization increased the percent BUSCO duplication by an average of only $1 \%$ for the smaller assembly, but by over $14 \%$ for the larger assembly. Given this, our recommendation is to employ digital normalization when the assembly is otherwise impossible, or when results are urgently needed, but that it should not be used by default for the production of transcriptome assemblies.

\subsection{Assembly}

\section{Summary Statement: Assemble your data using Trinity, then remove poorly supported contigs.}

For non-model organisms lacking reference genomic resources, the error correction, adapter and quality trimming reads should be assembled de novo into transcripts. Currently, the assembly package Trinity (Haas et al, 2013) is thought to currently be the most accurate (Li et all, 2014), and therefore is recommended over other assemblers. While attempting a merged assembly with multiple assemblers may ultimately result in the highest quality assembly, options for merging assemblies are currently limited, and therefore is not recommended.

Trinity's underlying algorithm has been pre-optimized to recover large numbers of alternative isoforms, 
including many that are minimally supported by read data. As a result, in many cases, the raw assembly will require filtration to remove these assembly artifacts. Reference dependent and independent evaluative tools (e.g., Transrate, BUSCO) allow for evidence-based post-assembly filtration. Typically, an initial quality-evaluation and filtration step is implemented using Transrate. This process assigns a score to the assembly, and creates an alternative assembly by removing contigs based on read-mapping metrics. This filtration step may result in the removal of a large proportion (as much as $67 \%$ ) of the transcripts.

Reference-based metrics are generated before and after this filtration step to ensure that filtration has not been too aggressive - that a significant number of known transcripts have not been removed. After Transrate filtration, or alternative to it, it is often helpful to employ a filtration step based on TPM. Because underlying assumptions of gene expression estimation software vary, which may result in variation of the actual estimates, gene expression is typically estimated using two different packages, Salmon and Kallisto. Transcripts whose abundance is less than either 1 or 0.5 are removed. Again, reference-based metrics are generated to ensure that a significant number of known transcripts are not removed.

The results of filtration on several datasets of varying size are presented in Figure 4. The reads used in the $1 \mathrm{M}, 5 \mathrm{M}, 10 \mathrm{M}, 20 \mathrm{M}$ subset assemblies were corrected with bfc, while the reads for the larger assemblies were corrected with RCorrector. Each dataset was trimmed to a quality of Phred $<2$, and assembled with Trinity. The raw assembly was filtered by Transrate and by gene expression. BUSCO evaluation was performed before and after these filtration steps. In general, for low coverage datasets (less than 20 million reads), filtering based on expression, using $\mathrm{TPM}=1$ as a threshold performs well, with Transrate filtering being too aggressive. With higher coverage data (more than 60 million reads) Transrate filtering may be optimal, as may gene expression filtering using a threshold of TPM=0.5. Again, the strength of this process is that it is guided by evidence, with filtering thresholds chosen based upon objective metrics. 
Complete BUSCO
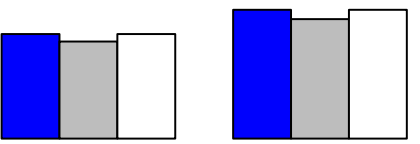
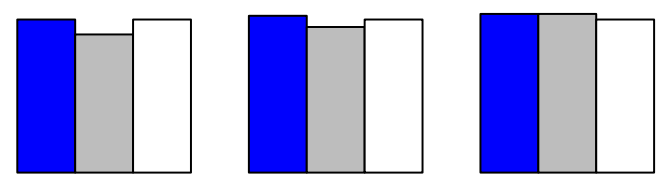

Number of Contigs

$\left.\begin{array}{rr} & 8 \\ & 8 \\ 0 & 0 \\ 0 & 0 \\ 0 & 1 \\ & 0\end{array}\right]$
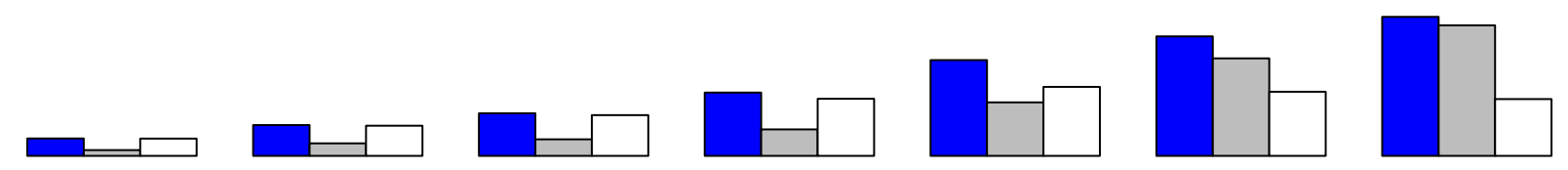

Size of assembly

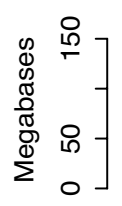

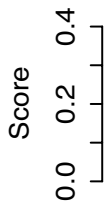

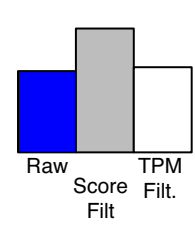

$1 \mathrm{M}$

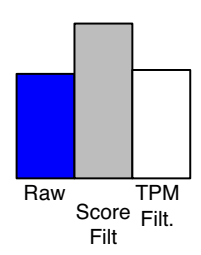

$5 \mathrm{M}$

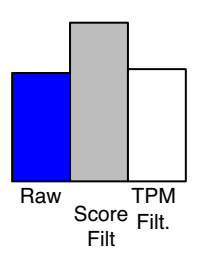

$10 \mathrm{M}$
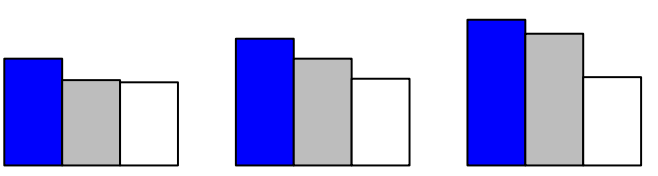

Transrate Score

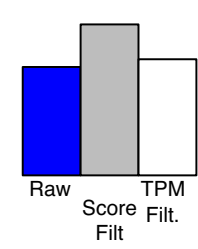

$20 \mathrm{M}$

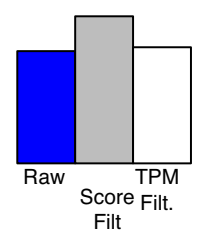

$40 \mathrm{M}$

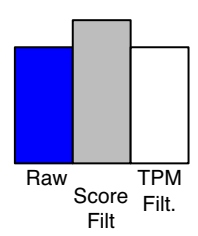

$60 \mathrm{M}$

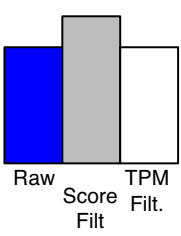

$80 \mathrm{M}$
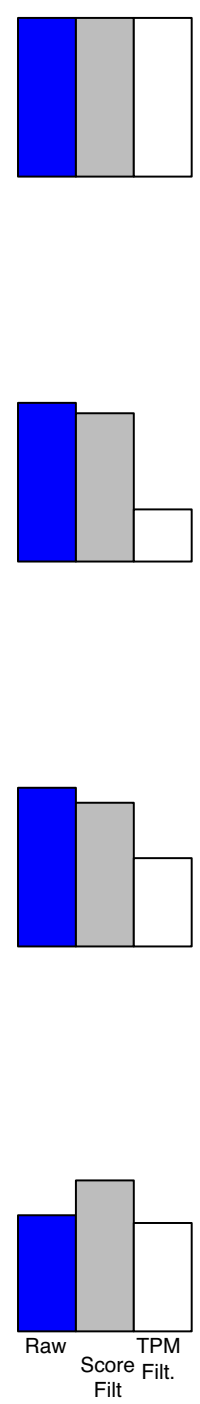

$100 \mathrm{M}$

Figure 4. Post-assembly filtration. Using assemblies from the 1M,5M,10M,20M,40M,60M,80M,100M read subsets, I evaluated the effects of Transrate and TPM filtration using a threshold of TPM=1. Both Transrate and TPM filtering reduced the number of contigs and assembly size, though the magnitudes were dependent on the depth of sequencing. BUSCO scores were either decreased in some cases, or stable in others, representing the differential effects of filtering on different sized assemblies. In general, for low coverage datasets (less than 20 million reads), filtering based on expression, using TPM=1 as a threshold performs well, with Transrate filtering being too aggressive. With higher coverage data (more than 60 million reads) Transrate filtering may perform better, as mat expression filtering with a lower threshold. 


\subsection{Annotation, post-assembly quality verification, \& reporting}

Summary Statement: Verify the quality of your assembly using content based metrics. Annotate using dammit Report Transrate score, BUSCO statistics, number of unique transcripts, etc. Do not report meaningless statistics such as N50.

Annotation is a critically important step in transcriptome assembly. Much like other steps, numerous options exist. Though the research requirements may drive the annotation process, I propose that a core set of annotations be provided with all de novo transcriptome assembly projects. The process through which these core annotations are accomplished is coordinated by the software package dammit. This software takes as input a fasta file and outputs a standard gff3 containing annotations. After annotation, but before downstream use, it is important to assess the quality of a transcriptome. Many authors have attempted to use typical genome assembly quality metrics for this purpose. In particular, N50 and other length-based summary statistic are often reported (e.g. (Hiz et al, 2014; Liang et al., 20133; Shinzato et al, 2014)). However, in addition to being a poor proxy for quality in genome assembly (Bradnam et all, 2013), N50 in the context of a transcriptome assembly carries very little information because the optimal contig length is not known (Li et al, 2014) - real transcripts vary greatly in length, ranging from tens of nucleotides to tens of thousands of nucleotides. Reportable metrics should be chosen based on their relevance for assembly optimization given the biological question at hand. In most cases, this means maximizing the number of transcripts that can be confidently attributed to the organism, while minimizing the number of technical artifacts related to the process of sequencing, quality control, and assembly. For many researchers, this means evaluation with both BUSCO and Transrate. The statistics found in Table 1 should be presented for all assemblies, with additional information supplementing these core vital statistics as needed.

\section{Testing the Oyster River Protocol}

To evaluate the Oyster River Protocol for Transcriptome Assembly, I selected three publicly available Illumina RNAseq datasets and their corresponding assembled transcriptomes. These three assemblies included the Nile Tilapia, Oreochromis niloticus ((Zhang et aI., 2013), SRR797490), an unpublished study of the Mediterranean black widow, Latrodectus tredecimguttatus (SRR954929), and lastly a work on Delia antiqua ((Gulo et aل, 2015), SRR916227). I analyzed the original transcriptomes using both BUSCO and Transrate, then followed the protocol as described here. Code for data analysis of the Oreochromis is 
available here. The other samples were processed in an identical fashion. The application of the Oyster River Protocol on these datasets resulted universally in a substantial (as much as $22 \%$ ) improvement in the completeness of assemblies. Given a major goal of these types of studies includes reconstruction all expressed genes, this improvement may have substantial improvement on downstream work. The Transrate score was dramatically improved as well, particularly in the Oreochromis and Delia assemblies. This improvement speaks to the improvement of the structure of the assembly.

The filtering process through which these more optimal assemblies were is key. Evaluating both the BUSCO and Transrate scores before and after, allows for an objective way to decide if filtering has been too restrictive or not. Indeed, for the Latrodectus assembly, both Transrate and TPM filtering reduced the BUSCO score, while substantially increasing the Transrate score. Depending on the goals of the experiment, it may be determined that the structural integrity of the assembly outweighs improved content. In contrast to how post-assembly filtering is typically done, this method allow for the researcher to make an informed decision about these processes.

\begin{tabular}{|c|c|c|c|c|c|}
\hline \multirow[b]{2}{*}{ Name } & \multicolumn{4}{|c|}{ Table 2} & \multirow[b]{2}{*}{ BUSCO Score } \\
\hline & Number Reads & Number Contigs & Assembly Size (Mb) & Transrate Score & \\
\hline Oreochromis & $25.2 \mathrm{M}$ & $79198 / 140035 / 100376 / 116038 / 88456$ & $32.0 / 75.1 / 69.5 / 58.6 / 57.7$ & $0.1103 / 0.2173 / 0.4778 / 0.2595 / 0.4479$ & $\mathrm{C}: 39 \%, \mathrm{M}: 46 \% / \mathrm{C}: 58 \% \mathrm{M}: 28 \% /$ \\
\hline Latrodectus & $27.6 \mathrm{M}$ & $10259 / 36394 / 30932 / 27973 / \mathrm{NA}$ & $10.6 / 13.5 / 13.1 / 10.9 / \mathrm{NA}$ & $0.43673 / 0.2795 / 0.4968 / 0.338 / \mathrm{NA}$ & $\mathrm{C}: 48 \%, \mathrm{M}: 38 \% / \mathrm{C}: 58 \%, \mathrm{M}: 28 \%$, \\
\hline Delia & $25.8 \mathrm{M}$ & $29451 / 49099 / 38614 / 46145 / 32689$ & $12.4 / 19.3 / 18.8 / 17.9 / 15.8$ & $0.393 / 0.2036 / 0.4572 / 0.2305 / 0.4341$ & $\mathrm{C}: 40 \%, \mathrm{M}: 48 \% / \mathrm{C}: 62 \%, \mathrm{M}: 21 \%$, \\
\hline
\end{tabular}

Table 2. The results of the application of the Oyster River Protocol to three available transcriptomes.

Within each column, the 5 metrics, separated by forward slashes are: 1 . The original assembly 2 . The raw Trinity assembly 3 . The Transrate filtered assembly 4 . The TPM=1 filtered assembly, and 5. The Transrate filtered assembly that has been further filtered by expression. In all cases the assembly content, as evaluated by the BUSCO score is dramatically improved over the original assembly. These content-improved assemblies have acceptable Transrate scores, which in 2 of 3 cases are vastly superior to the scores of the original assembly.

\section{Conclusions}

With the rapid adoption of high-throughput sequencing, studies of functional, evolutionary and population genomics are now being done by hundreds or even thousands of labs around the world. These studies typically begin with a de novo transcriptome assembly. Assembly may be accomplished in one of several different ways, using different software packages, with each method producing different results. This complexity begs the question - Which method(s) are optimal? Using reference and non-reference based evaluative methods, I have proposed a set of guidelines The Oyster River Protocol for Transcriptome Assembly that aim to standardize and facilitate the process of transcriptome assembly. These 
recommendations include limiting assembly to between 20 million and 40 million sequencing reads from single individual where possible, error correction of reads, gently quality trimming, assembly filtering using Transrate or gene expression, annotation using dammit, and appropriate reporting. The processes result in a high quality transcriptome assembly appropriate for downstream usage.

\section{Acknowledgments}

This work was significantly improved by discussions with Richard Smith-Unna, Brian Haas and many others. More generally, the work and it's presentation has been influenced by supporters of the Open Access and Science movements.

\section{References}

Alameldin, H.F., Awad, S., Boucher, E., Caldwell, A., Cartwright, R., Charbonneau, A., Constantinides, B., Fay, S., Fenton, J., Fenzl, T., Fish, J., Garcia-Gutierrez, L., Garland, P., Gluck, J., González, I., Guermond, S., Guo, J., Gupta, A., Herr, J.R., Howe, A., Hyer, A., Härpfer, a., Irber, L., Kidd, R., Lin, D., Lippi, J., Mansour, T., McA’Nulty, P., Mizzi, J., Murray, K.D., Nahum, J.R., Nanlohy, K., Nederbragt, A.J., Ortiz-Zuazaga, H., Ory, J., Pell, J., Pepe-Ranney, C., Russ, Z.N., Schwarz, E., Seaman, J., Sievert, S., Simpson, J., Skennerton, C.T., Spencer, J., Srinivasan, R., Standage, D., Stapleton, J.A., Steinman, S.R., Stein, J., Taylor, B., Trimble, W., Wiencko, H.L., Wright, M., Wyss, B., Zhang, Q., Zyme, E., 2015. The khmer software package: enabling efficient nucleotide sequence analysis. F1000Research 4, 900.

Au, K.F., Sebastiano, V., Afshar, P.T., Durruthy, J.D., Lee, L., Williams, B.A., van Bakel, H., Schadt, E.E., Reijo-Pera, R.A., Underwood, J.G., Wong, W.H., 2013. Characterization of the human ESC transcriptome by hybrid sequencing. PNAS 110, 201320101-30.

Bolger, A.M., Lohse, M., Usadel, B., 2014. Trimmomatic: a flexible trimmer for Illumina sequence data. Bioinformatics 30, btu170-2120.

Bradnam, K.R., Fass, J.N., Alexandrov, A., Baranay, P., Bechner, M., Birol, I., Boisvert, S., Chapman, J.A., Chapuis, G., Chikhi, R., Chitsaz, H., Chou, W.C., Corbeil, J., Del Fabbro, C., Docking, T.R., Durbin, R., Earl, D., Emrich, S., Fedotov, P., Fonseca, N.A., Ganapathy, G., Gibbs, R.A., Gnerre, S., Godzaridis, E., Goldstein, S., Haimel, M., Hall, G., Haussler, D., Hiatt, J.B., Ho, I.Y., Howard, J., Hunt, M., Jackman, S.D., Jaffe, D.B., Jarvis, E.D., Jiang, H., Kazakov, S., Kersey, P.J., Kitzman, J.O., Knight, J.R., Koren, 
S., Lam, T.W., Lavenier, D., Laviolette, F., Li, Y., Li, Z., Liu, B., Liu, Y., Luo, R., Maccallum, I., MacManes, M.D., Maillet, N., Melnikov, S., Naquin, D., Ning, Z., Otto, T.D., Paten, B., Paulo, O.S., Phillippy, A.M., Pina-Martins, F., Place, M., Przybylski, D., Qin, X., Qu, C., Ribeiro, F.J., Richards, S., Rokhsar, D.S., Ruby, J.G., Scalabrin, S., Schatz, M.C., Schwartz, D.C., Sergushichev, A., Sharpe, T., Shaw, T.I., Shendure, J., Shi, Y., Simpson, J.T., Song, H., Tsarev, F., Vezzi, F., Vicedomini, R., Vieira, B.M., Wang, J., Worley, K.C., Yin, S., Yiu, S.M., Yuan, J., Zhang, G., Zhang, H., Zhou, S., Korf, I.F., 2013. Assemblathon 2: evaluating de novo methods of genome assembly in three vertebrate species. GigaScience 2, 10.

Bray, N., Pimentel, H., Melsted, P., Pachter, L., 2015. Near-optimal RNA-Seq quantification. arXiv.org arXiv: $14054600 / 472655 b 5258$

Brown, C.T., Howe, A., Zhang, Q., Pyrkosz, A.B., Brom, T.H., 2012. A Reference-Free Algorithm for Computational Normalization of Shotgun Sequencing Data. arXiv.org arXiv:1203.4802v2.

Cox, M.P., Peterson, D.A., Biggs, P.J., 2010. SolexaQA: At-a-glance quality assessment of Illumina second-generation sequencing data. BMC Bioinformatics 11, 485.

Fitzpatrick, M., Ben-Shahar, Y., Vet, L., Smid, H., Robinson, G.E., Sokolowski, M., 2005. Candidate genes for behavioural ecology. Trends In Ecology \& Evolution 20, 96-104.

Garber, M., Grabherr, M.G., Guttman, M., Trapnell, C., 2011. Computational methods for transcriptome annotation and quantification using RNA-seq. Nature Methods 8, 469-477.

Glenn, T.C., 2011. Field guide to next-generation DNA sequencers. Molecular Ecology Resources 11, $759-769$.

Guo, Q., Hao, Y.J., Li, Y., Zhang, Y.J., Ren, S., Si, F.L., Chen, B., 2015. Gene cloning, characterization and expression and enzymatic activities related to trehalose metabolism during diapause of the onion maggot Delia antiqua (Diptera: Anthomyiidae). Gene 565, 106-115.

Haas, B.J., Papanicolaou, A., Yassour, M., Grabherr, M., Blood, P.D., Bowden, J., Couger, M.B., Eccles, D., Li, B., Lieber, M., MacManes, M.D., Ott, M., Orvis, J., Pochet, N., Strozzi, F., Weeks, N., Westerman, R., William, T., Dewey, C.N., Henschel, R., Leduc, R.D., Friedman, N., Regev, A., 2013. De novo transcript sequence reconstruction from RNA-seq using the Trinity platform for reference generation and analysis. Nature Protocols 8, 1494-1512. 
Han, H., Irimia, M., Ross, P.J., Sung, H.K., Alipanahi, B., David, L., Golipour, A., Gabut, M., Michael, I.P., Nachman, E.N., Wang, E., Trcka, D., Thompson, T., O’Hanlon, D., Slobodeniuc, V., Barbosa-Morais, N.L., Burge, C.B., Moffat, J., Frey, B.J., Nagy, a., Ellis, J., Wrana, J.L., Blencowe, B.J., 2013. MBNL proteins repress ES-cell-specific alternative splicing and reprogramming. Nature 498, 241-245.

Heo, Y., Wu, X.L., Chen, D., Ma, J., Hwu, W.M., 2014. BLESS: Bloom filter-based error correction solution for high-throughput sequencing reads. Bioinformatics 30, 1354-1362.

Hiz, M.C., Canher, B., Niron, H., Turet, M., 2014. Transcriptome analysis of salt tolerant common bean (Phaseolus vulgaris L.) under saline conditions. PLOS ONE 9, e92598.

Iqbal, Z., Caccamo, M., Turner, I., Flicek, P., McVean, G., 2012. De novo assembly and genotyping of variants using colored de Bruijn graphs. Nature Publishing Group 44, 226-232.

Jiang, H., Lei, R., Ding, S.W., Zhu, S., 2014. Skewer: a fast and accurate adapter trimmer for next-generation sequencing paired-end reads. BMC Bioinformatics 15, 182.

Johnson, J.M., Castle, J., Garrett-Engele, P., Kan, Z., Loerch, P.M., Armour, C.D., Santos, R., Schadt, E.E., Stoughton, Roland Shoemaker, D.D., 2003. Genome-wide survey of human alternative pre-mRNA splicing with exon junction microarrays. Science 302, 2141-2144.

Le, H.S., Schulz, M.H., McCauley, B.M., Hinman, V.F., Bar-Joseph, Z., 2013. Probabilistic error correction for RNA sequencing. Nucleic Acids Research 41, 1-11.

Li, B., Fillmore, N., Bai, Y., Collins, M., Thomson, J.A., Stewart, R., Dewey, C.N., 2014. Evaluation of de novo transcriptome assemblies from RNA-Seq data. Genome Biology 15, 663-21.

Li, H., 2015. BFC: correcting Illumina sequencing errors. Bioinformatics 31, 2885-2887.

Liang, C., Liu, X., Yiu, S.M., Lim, B.L., 2013. De novo assembly and characterization of Camelina sativa transcriptome by paired-end sequencing. BMC Genomics 14, 146.

Love, M.I., Huber, W., anders, S., 2014. Moderated estimation of fold change and dispersion for RNA-seq data with DESeq2. Genome Biology 15, 550.

MacManes, M.D., 2014. On the optimal trimming of high-throughput mRNA sequence data. Frontiers in Genetics 5 .

MacManes, M.D., Eisen, M.B., 2013. Improving transcriptome assembly through error correction of high-throughput sequence reads. PeerJ 1, e113. 
MacManes, M.D., Lacey, E.A., 2012. The Social Brain: Transcriptome Assembly and Characterization of the Hippocampus from a Social Subterranean Rodent, the Colonial Tuco-Tuco (Ctenomys sociabilis). PLOS ONE 7, e45524.

Martin, M., 2011. Cutadapt removes adapter sequences from high-throughput sequencing reads. EMBnet.journal 17, pp. 10-12.

McDonald, E., Brown, C.T., 2013. khmer: Working with Big Data in Bioinformatics. arXiv.org

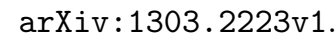

Modrek, B., Resch, A., Grasso, C., Lee, C., 2001. Genome-wide detection of alternative splicing in expressed sequences of human genes. Nucleic Acids Research 29, 2850-2859.

Panhuis, T.M., 2006. Molecular evolution and population genetic analysis of candidate female reproductive genes in Drosophila. Genetics 173, 2039-2047.

Patro, R., Duggal, G., Kingsford, C., 2015. Accurate, fast, and model-aware transcript expression quantification with Salmon. biorxiv.org, 1-35.

Pell, J., Hintze, A., Canino-Koning, R., Howe, A., Tiedje, J.M., Brown, C.T., 2012. Scaling metagenome sequence assembly with probabilistic de Bruijn graphs. Proceedings of the National Academy of Sciences 109, 13272-13277.

Robinson, M.D., McCarthy, D.J., Smyth, G.K., 2010. edgeR: a Bioconductor package for differential expression analysis of digital gene expression data. Bioinformatics 26, 139-140.

Shinzato, C., Inoue, M., Kusakabe, M., 2014. A snapshot of a coral "holobiont": a transcriptome assembly of the scleractinian coral, porites, captures a wide variety of genes from both the host and symbiotic zooxanthellae. PLOS ONE 9, e85182.

Simão, F.A., Waterhouse, R.M., Ioannidis, P., Kriventseva, E.V., Zdobnov, E.M., 2015. BUSCO: assessing genome assembly and annotation completeness with single-copy orthologs. Bioinformatics 31, 3210-3212.

Simpson, J.T., Durbin, R., 2012. Efficient de novo assembly of large genomes using compressed data structures. Genome Research 22, 549-556.

Smith-Unna, R.D., Boursnell, C., Patro, R., Hibberd, J.M., Kelly, S., 2015. TransRate: reference free quality assessment of de novo transcriptome assemblies. bioRxiv URL:

http://biorxiv.org/content/early/2015/06/27/021626. 
Song, L., Florea, L., 2015. Rcorrector: efficient and accurate error correction for Illumina RNA-seq reads. GigaScience 4, 48.

Song, L., Florea, L., Langmead, B., 2014. Lighter: fast and memory-efficient sequencing error correction without counting. Genome Biology 15, 509.

Studholme, D.J., 2010. De novo assembly of short sequence reads. Briefings In Bioinformatics 11, 457-472.

Vijay, N., Poelstra, J.W., Künstner, A., Wolf, J.B.W., 2013. Challenges and strategies in transcriptome assembly and differential gene expression quantification. A comprehensive in silico assessment of RNA-seq experiments. Molecular Ecology 22, 620-634.

Wolf, J.B.W., 2013. Principles of transcriptome analysis and gene expression quantification: an RNA-seq tutorial. Molecular Ecology Resources 13, 559-572.

Xie, Y., Wu, G., Tang, J., Luo, R., Patterson, J., Liu, S., Huang, W., He, G., Gu, S., Li, S., Zhou, X., Li, Y., Xu, X., Wong, G.K.S., Wang, J., 2014. SOAPdenovo-Trans: de novo transcriptome assembly with short RNA-Seq reads. Bioinformatics 30, 1660-1666.

Zhang, R., Zhang, L.l., Ye, X., Tian, Y.y., Sun, C.f., Lu, M.x., Bai, J.j., 2013. Transcriptome profiling and digital gene expression analysis of Nile tilapia (Oreochromis niloticus) infected by Streptococcus agalactiae. Molecular biology reports 40, 5657-5668. 